\title{
INTEGER AND CONSTRAINT PROGRAMMING APPROACHES FOR PROVIDING OPTIMALITY TO THE BANDWIDTH MULTICOLORING PROBLEM
}

\author{
Bruno Dias ${ }^{1, *}$, Rosiane de Freitas ${ }^{1}$, Nelson Maculan $^{2}$ and Philippe Michelon ${ }^{3}$
}

\begin{abstract}
In this paper, constraint and integer programming techniques are applied to solving bandwidth coloring problems, in the sense of proving optimality or finding better feasible solutions for benchmark instances from the literature. The Bandwidth Coloring Problem (BCP) is a generalization of the classic vertex coloring problem (VCP), where, given a graph, its vertices must be colored such that not only adjacent ones do not share the same color, but also their colors must be separated by a minimum given value. BCP is further generalized to the Bandwidth Multicoloring Problem (BMCP), where each vertex can receive more than one different color, also subject to separation constraints. BMCP is used to model the Minimum Span Channel Assignment Problem (MS-CAP), which arises in the planning of telecommunication networks. Research on algorithmic strategies to solve these problems focus mainly on heuristic approaches and the performance of such methods is tested on artificial and real scenarios benchmarks, such as GEOM, Philadelphia and Helsinki sets. We achieve optimal solutions or provide better upper bounds for these well-known instances, We also compare the effects of multicoloring demands on the performance of each exact solution approach, based on empirical analysis.
\end{abstract}

Mathematics Subject Classification. 90C10, 68R10, 90C27.

Received December 31, 2018. Accepted June 17, 2020.

\section{INTRODUCTION}

Let $G=(V, E)$ be an undirected graph. A $k$-coloring of $G$ is an assignment of colors $\{1,2, \ldots, k\}$ to the vertices of $G$ so no two adjacent vertices share the same color. The chromatic number $\chi_{G}$ of a graph is the minimum value of $k$ for which $G$ is $k$-colorable. The classic vertex coloring problem (VCP), which consists in finding the chromatic number of a graph, is a well-known combinatorial optimization problem which belongs to NP-hard complexity class $[10,12,14]$.

One of the main applications of such problems consists of assigning channels to transmitters in a mobile wireless network. Each transmitter is responsible for the calls made in the area it covers and the communication among devices is made through a channel consisting of a discrete slice of the electromagnetic spectrum. However,

Keywords. Bandwidth coloring, channel assignment, integer and constraint programming, graph theory.

1 Instituto de Computação (IComp/UFAM), Universidade Federal do Amazonas, Manaus, Brazil.

2 PESC/COPPE, Universidade Federal do Rio de Janeiro (UFRJ), Rio de Janeiro - RJ, Brazil.

3 Centre d'Enseignement et de Recherche en Informatique, Université d'Avignon et des Pays de Vaucluse, Avignon, France.

*Corresponding author: bruno.dias@icomp.ufam.edu.br 

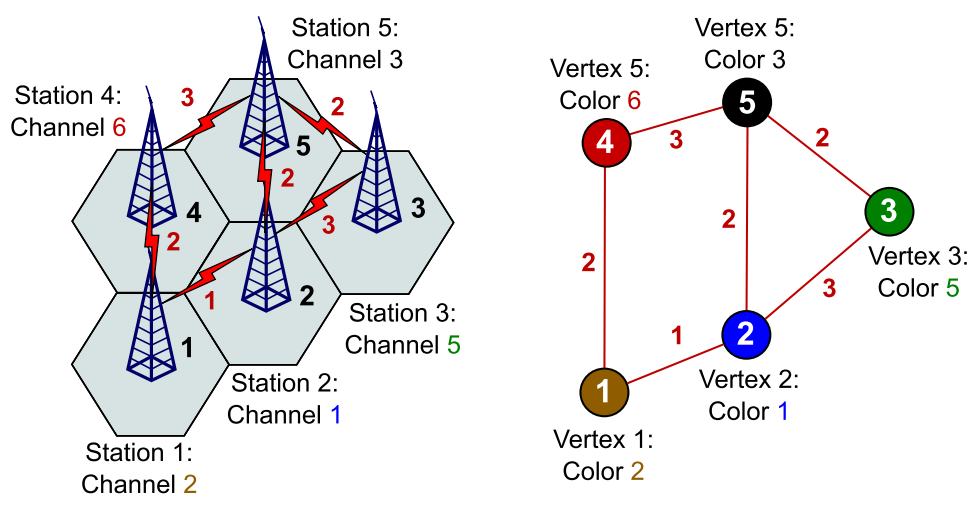

FiguRE 1. Example of channel assignment and its modeling as an instance of Bandwidth Coloring Problem.

the channels cannot be assigned to calls in an arbitrary way, since there is the problem of interference among devices located near each other using approximate channels. Given this scenario, channels must be assigned to calls so interference is avoided and the spectrum usage is minimized [17,27,28].

Thus, the channel assignment scenario can be modeled as a graph coloring problem by considering each transmitter as a vertex in a simple undirected graph and the channels to be assigned as the colors the vertices will receive. Some more general graph coloring problems were proposed in the literature in order to take the separation among channels into account, such as the T-coloring problem, also known as the Generalized Coloring Problem (GCP) $[8,13]$, which was one of the first combinatorial optimization approaches to channel assignment, where each edge is assigned a given forbidden set such that the absolute difference between colors given to each incident edge is in the set. The scenario where stations may need more than one channel, that is, vertices in the corresponding graph may have a demand of more than one color, is modeled as the set T-coloring problem [8], in which there is also a forbidden set for each vertex so that the absolute difference between colors assigned to the same vertex must not be in its forbidden set.

A special case of T-coloring consists of forbidden sets containing only consecutive integer numbers starting from zero (that is, sets of form $\{0,1,2, \ldots, d\}$, or, equivalently, intervals $[0, \ldots, d] \subset \mathbb{Z}$ ), which means the absolute difference between colors assigned to each vertex must be greater or equal a certain value. This case is known in the literature as the Bandwidth Coloring Problem (BCP) [18, 20,21,24], since this requirement occurs with respect to frequency bands in a wireless network. An example of channel assignment instance and its corresponding model as a BCP instance is shown in Figure 1. Note that the edges are only assigned the upper interval bound $d_{i, j}$.

The separation constraints in the $\mathrm{BCP}$ can be seen as a type of distance constraint, so we can see the channel assignment as a type of distance geometry (DG) problem, since we have to place the channels in the transmitters respecting some distances imposed in the edges, where a coloring $x: V \rightarrow \mathbb{N}$, such that $\forall\{i, j\} \in E,|x(i)-x(j)| \geq d_{i, j}$, must be found [6,7]. This theoretical model can be used to derive integer and constraint programming models based on characteristics from the problem as well as previous works with similar problems.

The main contribution of this paper consists of the use of integer and constraint programming models to provide exact solutions to $\mathrm{BCP}$, applying them to existing instances, including ones based on real channel assignment scenarios. There are many algorithms for BCP in the literature, including some based on classic metaheuristics, including simulated annealing [3], local search [9], evolutionary algorithms [20] and tabu search $[8,18]$. However, there is no optimality guarantee in these methods. Using integer and constraint programming approaches, we were able to prove the optimality of some solutions found by heuristic methods, such as 
the multistart iterated tabu search proposed in [18], and obtain better upper bounds for some problems, including optimal solutions for open instances. In this process, we also found some inconsistencies in the literature with respect to the quality of some approximate algorithms, where the heuristic presented solutions better than the optimal ones found by an exact method.

The remainder of this paper is organized as follows. Section 2 formally defines the Bandwidth Coloring Problem and discusses its characteristics. Section 3 gives a mathematical formulation in constraint programming based on theoretical distance geometry models, and also gives an integer programming formulation for comparison. Section 4 shows results of some experiments done with implementations of the mathematical models. Finally, in Section 5, final remarks are made and next steps of ongoing research are stated.

\section{Bandwidth Coloring Definitions AND MODEls}

The Bandwidth Coloring Problem (BCP) can be stated as follows. Given an undirected graph $G=(V, E)$ where, for each edge $(i, j) \in E$, there is a positive integer $d_{i, j}$, each vertex $i$ must receive a color $x(i)$ and, for each edge $(i, j) \in E$, the condition $|x(i)-x(j)| \geq d_{i, j}$ must hold.

This problem is a special case of T-coloring [13], since we can build a T-coloring instance from any BCP instance by setting the forbidden set of an edge $(i, j) \in E$ to $T_{i, j}=\left\{0,1, \ldots, d_{i, j}\right\}$, The constraint $|x(i)-x(j)| \notin T_{i, j}$ is, then, the same as the one from BCP, that is, the former corresponds to a T-coloring instance with forbidden sets consisting of consecutive values.

The constraints imposed on BCP are a kind of distance constraint, so it can be modeled using concepts from distance geometry (DG) [4-6]. The fundamental Distance Geometry Problem (DGP) has, as input, a graph $G=(V, E)$ such that for each edge $(i, j) \in E$, a distance $d_{i, j} \in \mathbb{R}_{+}$is attributed to it and an embedding $x: V \rightarrow \mathbb{R}^{n}$ must be found such that $\|x(i)-x(j)\|=d_{i, j}$ for all $(i, j) \in E$. When $n=1$, we have 1-DGP, which is equivalent to 1-Embeddability [25]. If the condition $\forall(i, j) \in E, d_{i, j} \in \mathbb{N}$ also holds, then we have the Discretizable Distance Geometry Problem (DDGP) in one dimension, or 1-DDGP. Based on this model, we can formalize the following coloring problem.

Definition 2.1 (Minimum Equal Coloring Distance Geometry Problem (MinEQ-CDGP)). Given a simple weighted undirected graph $G=(V, E)$, where, for each $(i, j) \in E$, there is a weight $d_{i, j} \in \mathbb{N}$, find an embedding $x: V \rightarrow \mathbb{N}$ such that $|x(i)-x(j)|=d_{i, j}$ for each $(i, j) \in E$ whose span $S$, defined as $S=\max _{i \in V} x(i)$, that is, the maximum used color, is the minimum possible.

By substituting the equality constraint in MinEQ-CDGP into an inequality (greater than or equal) and considering only one dimension for the embedding and only its positive integer points, a DG model for BCP can be derived, as defined below.

Definition 2.2 (Minimum Greater than or Equal Coloring Distance Geometry Problem - MinGEQ-CDGP $[4,5])$. Given a simple weighted undirected graph $G=(V, E)$, where, for each $(i, j) \in E$, there is a weight $d_{i, j} \in \mathbb{N}$, find an embedding $x: V \rightarrow \mathbb{N}$ such that $|x(i)-x(j)| \geq d_{i, j}$ for each $(i, j) \in E$ whose $\operatorname{span}\left(\max _{i \in V} x(i)\right)$ is the minimum possible.

The MinGEQ-CDGP is equivalent to BCP, since, for each vertex $i \in V$, the point $x(i)$ assigned to it in MinGEQ-CDGP corresponds to its color in BCP. A special case when, for all $(i, j) \in E$, we have $d_{i, j}=\beta$, where $\beta \in \mathbb{N}$. When $\beta=1$, we have a VCP instance, where colors between adjacent vertices must only be different among each other. The input graph can be stated only by its vertices, edges and the $\beta$ value. The corresponding DG model is stated below and exemplified in Figure 2.

Definition 2.3 (Minimum Greater than or Equal Coloring Distance Geometry Problem with Constant Distances - MinGEQ-CDGP-Unif). [4,5]: Given a simple weighted undirected graph $G=(V, E)$, and a nonnegative integer $\beta$, find an embedding $x: V \rightarrow \mathbb{N}$ such that $|x(i)-x(j)| \geq \beta$ for each $(i, j) \in E$ whose $\operatorname{span}\left(\max _{i \in V} x(i)\right)$ is the minimum possible. 


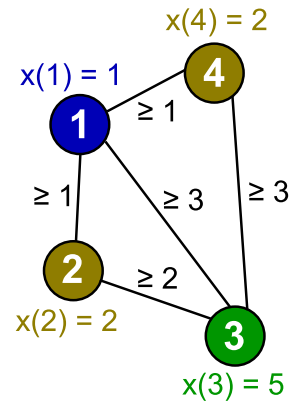

(a) MinGEQ-CDGP.

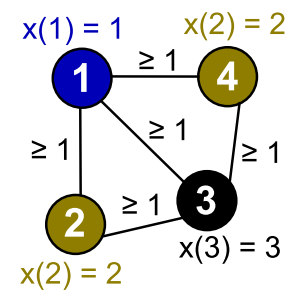

(b) MinGEQ-CDGP-Unif.

Figure 2. Examples of instances for MinGEQ-CDGP (BCP) and MinGEQ-CDGP-Unif problems. Note that the MinGEQ-CDGP-Unif instance used is also a VCP one.

A variation of $\mathrm{BCP}$ is the Bandwidth Multicoloring Problem (BMCP), which is also a special case of set T-coloring, where the vertex $i$ has an associated color demand $q_{i}$ and a weight $d_{i, i}$, such that it receives $q_{i}$ colors (instead of just one). Let $x(i, k)$ be the $k$-th color assigned to vertex $i$ (with $1 \leq k \leq q_{i}$ ). Then, for each pair of colors $x(i, k)$ and $x(i, m)$ associated to $i$, the constraint $|x(i, k)-x(i, m)| \geq d_{i, i}$ must be respected in BMCP. An equivalent problem to BMCP is the Minimum Span Channel Assignment (MS-CAP) [1,17], also known as Minimum Span Frequency Assignment (MS-FAP) where channels correspond to colors and devices to vertices. However, the input consists of positions for these devices and reuse distances, where, based on the distance between two devices, the separation between colors is calculated. If this input is converted into a graph where edges are weighted according to this separation, then it becomes a BMCP instance.

For BMCP, we can extend MinGEQ-CDGP as shown below.

Definition 2.4 (Minimum Greater than or Equal Multicoloring Distance Geometry Problem - MinGEQ-MultiCDGP). Given a simple weighted undirected graph $G=(V, E)$, where, for each $(i, j) \in E$, there is a weight $d_{i, j} \in \mathbb{N}$, and, for each vertex $i \in V$, there are weights $q_{i}, d_{i, i} \in \mathbb{N}$ find an embedding $x: V \rightarrow 2^{\mathbb{N}}$ such that $|x(i)|=q_{i}$; letting $x(i, k)$ be the $k$-th color assigned to $i$ then for each $(i, j) \in E, 1 \leq k \leq q_{i}$ and $1 \leq m \leq q_{j},|x(i, k)-x(j, m)| \geq d_{i, j}$; and, for each $i \in V, 1 \leq k, l \leq q_{i},|x(i, k)-x(i, l)| \geq d_{i, i}$; whose span $\left(\max _{i \in V}, 1 \leq k \leq q_{i} x(i)\right)$ is the minimum possible.

As is the case with all multicoloring problems, there is an equivalence between MinGEQ-CDGP and MinGEQMulti-CDGP, that is, an instance of MinGEQ-Multi-CDGP can be converted into MinGEQ-CDGP by replicating each vertex $i$ into a clique of $q_{i}$ subvertices. Each edge in the clique has a distance imposed on it equal to $d_{i, i}$ from the original MinGEQ-Multi-CDGP instance, and each subvertex is adjacent to all other vertices that the original vertex also was adjacent to. Figure 3 shows an example of this conversion. By employing this transformation, any algorithm for MinGEQ-CDGP can be used for MinGEQ-Multi-CDGP. However, if the algorithm does not explore specific characteristics of multicoloring, its runtime will be much higher $[18,22]$.

In Figure 4, we show the hierarchy of coloring problems shown in this section and which ones are the focus of this paper. We refer the reader to [5] for more information about other distance coloring models.

\section{Constraint And integer programming approaches}

The distance geometry approach for graph coloring problems with distances is directly mapped to a constraint programming (CP) approach, since it addresses the graph coloring problem with distances as an embedding of the graph in one dimension, that is, a labeling of the vertices with natural numbers indicating its projection on the line, such that the distances of the segments defined by the edge weights of the graph are met, and such 


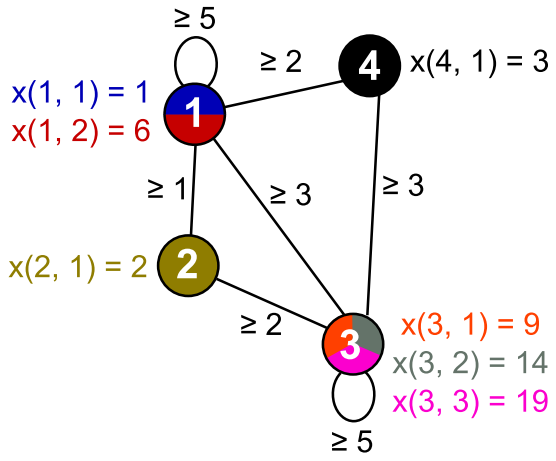

(a) Original MinGEQ-Multi-CDGP instance.

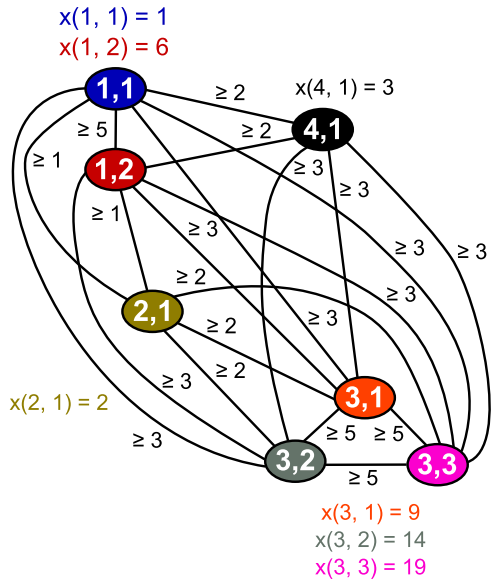

(b) Transformation to MinGEQCDGP.

Figure 3. Example of MinGEQ-Multi-CDGP instance and its transformation into a MinGEQCDGP instance.

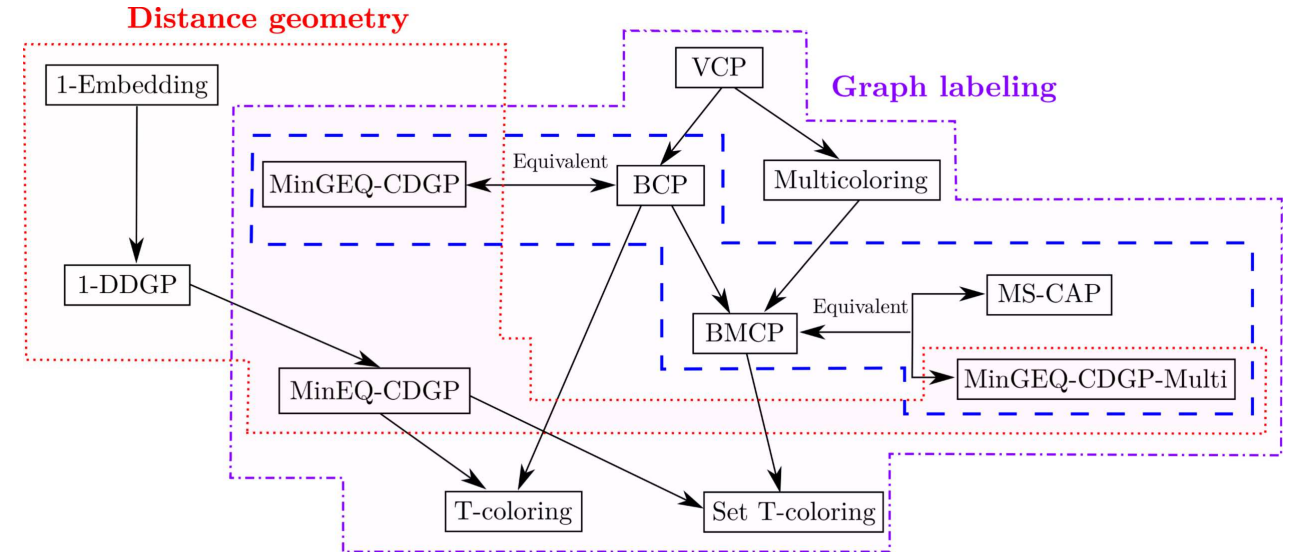

FiguRE 4. Hierarchy of graph coloring problems with distance constraints. The problems explored in this paper are highlighted by the dashed polygon.

that the total range is minimized (the span of colors is minimized). In this way, a mathematical technique that handles these segments or distance constraints is very useful, which is the case for the constraint programming model. We also present a traditional integer programming formulation, to compare both approaches.

Thus, the first formulation presented is based on constraint programming, which will be denoted by MinGEQCDGP-CP and is proposed in this work by directly using the problem definition.

Let $x_{i}$ be an integer variable consisting of the color assigned to vertex $i$. Then MinGEQ-CDGP-CP is defined as:

$$
\begin{array}{cll}
\text { Minimize } & \max _{i \in V} x(i) & \\
\text { Subject to } & |x(i)-x(j)| \geq d_{i, j} & (\forall(i, j) \in E) \\
& x(i) \in \mathbb{Z}^{*} & (\forall i \in V)
\end{array}
$$


The objective (3.1) is to minimize the maximum used color among all vertices (the coloring span). Constraint set (3.2) involves weighted edges with inequality constraints. For each variable $x_{i}$, the initial domain (before constraint propagation) $D\left(x_{i}\right)$ consists of all integers between 1 and a given upper bound $U$, that is, $D\left(x_{i}\right)=[1, \ldots, U]$. Note that all initial domains are the same. This model has $O(|V|)$ variables (one for each vertex) and $O(|E|)$ constraints (one for each edge), since it captures the essential definition of the problem.

A special CP model can be stated for the case when all distances are the same (MinGEQ-CDGP-Unif) and the input graph is complete, using a specific global constraint. We propose such model, denoted by MinGEQCDGP-Unif-CP, which is defined as:

$$
\begin{array}{cl}
\text { Minimize } & \max _{i \in V} x(i) \\
\text { Subject to } & \text { allMinDistance }(\{x(i): i \in V\}, \beta) \\
& x(i) \in \mathbb{Z}^{*}
\end{array}
$$

The allMinDistance global constraint used in (3.5) takes as its arguments a set of variables and a number $w$, which is the minimum distance to be respected, and ensures that, for all pairs of variables $y$ and $z$ in the set, the condition $|y-z| \geq w$ is valid, which is the case for each vertex and its neighbors. This special case has $O(|V|)$ variables and only one global constraint. This formulation has fewer constraints and is able to use specialized propagators. We note that allMinDistance is a generalization of the classical allDifferent global constraint, such that when the constant $w$ (that is, the distance to be respected) is set to 1 , the result of both constraints would be the same. However, there is no guarantee that the algorithms for separating allDifferent will be used in this scenario.

For MinGEQ-Multi-CDGP, a CP formulation can be constructed by using characteristics from both previously shown models. As discussed in Section 2, a multicoloring problem can be converted into a coloring with single demands by transforming a vertex $i$ into a clique of $q_{i}$ vertices, each adjacent to all other vertices that were adjacent to $i$. By using this, we have that, essentially, each vertex consists of a small MinGEQ-CDGPUnif subinstance, where the larger graph (that is, considering the constraints imposed on the original edges between different vertices), if its multicoloring demands are ignored, is a MinGEQ-CDGP instance. Based on this combination, we propose the following CP formulation, denoted by MinGEQ-Multi-CDGP-CP:

$$
\begin{array}{cll}
\text { Minimize } & \max _{\substack{i \in V \\
1 \leq k \leq q_{i}}} x(i, k) & \\
\text { Subject to } & |x(i, k)-x(j, m)| \geq d_{i, j} & \left(\forall(i, j) \in E, 1 \leq k \leq q_{i}\right) \\
& & \left.1 \leq m \leq q_{j}\right) \\
& \text { allMinDistance }\left(\left\{x(i, k): 1 \leq k \leq q_{i}\right\}, d_{i, i}\right) & (\forall i \in V) \\
& x(i, k) \in \mathbb{Z}^{*} & \left(\forall i \in V, 1 \leq k \leq q_{i}\right)
\end{array}
$$

In MinGEQ-Multi-CDGP-CP, constraints (3.8) ensure that colors assigned to different vertices respect the distance imposed on edges. Constraints (3.9) require that different colors assigned to the same vertex respect the minimum distance $d_{i, i}$ between each other (using the allMinDistance global constraint, since they form a small MinGEQ-CDGP-Unif subinstance). This formulation has $O\left(|V| q_{\max }\right.$ ) variables (where $q_{\max }=\max _{i \in V} q_{i}$, that is, the largest color demand in the graph), since, for each color needed to each vertex, there is a variable, and $O\left(|E| q_{\max }\right)$ constraints.

The second mathematical formulation approach is an integer programming model, which will be denoted by MinGEQ-CDGP-IP. It is based on models defined in [17], where we modified the formulation in order to make it more compact. A similar formulation was proposed independently in [19]. Two sets of variables are used:

$$
\begin{aligned}
& -x_{i c}= \begin{cases}1 & \text { if color } c \text { is assigned to vertex } i \\
0 & \text { otherwise. }\end{cases} \\
& -z_{\max }=\text { value of maximum color used in the solution (the coloring span). }
\end{aligned}
$$


Using these variables, MinGEQ-CDGP-IP is defined as follows:

$$
\begin{array}{lll}
\text { Minimize } & z_{\max } & \\
\text { Subject to } & \sum_{c=1}^{U} x_{i c}=1 & (\forall i \in V) \\
& x_{i c}+x_{j e} \leq 1 & \left(\forall(i, j) \in E ; 1 \leq c, e \leq U|| c-e \mid<d_{i, j}\right) \\
& z_{\max } \geq c x_{i c} & (\forall i \in V ; 1 \leq c \leq U) \\
x_{i c} \in\{0,1\} & (\forall i \in V ; 1 \leq c \leq U) \\
& z_{\max } \in \mathbb{R} &
\end{array}
$$

In MinGEQ-CDGP-IP, the objective (3.11) is to minimize the value of variable $z_{\max }$, which will be the coloring span. Constraint set (3.12) ensures that all vertices must be colored. Constraint set (3.13) ensures that the absolute difference between the colors assigned to adjacent vertices is less than the distance given by the weight of the respective edge, then only one of the vertices can receive that color. Constraints (3.14) require that variable $z_{\max }$ be greater than or equal to any color used, so it will be the maximum color used. Constraints (3.15) require that variables in the set $x_{i c}$ use only values 0 and 1 , while constraint (3.16) states that $z_{\max }$ is a free variable, although its value will always be an integer, since, at the optimal solution, $z_{\max }=c x_{i c}$ for some $i \in V$ and $c \in \llbracket 1, U \rrbracket$. The value $U$ denotes the upper bound for colors to be used, since variables are indexed by vertex and color, so the color set must be limited. This IP model has $O(U|V|$ ) variables (one for each pair of color and vertex) and $O\left(U|V|+U^{2}|E|\right)$ constraints, that is, it has pseudopolynomial size.

For MinGEQ-Multi-CDGP, the integer programming model can also be changed to accomodate multicoloring demands. Such modified formulation, which will be denoted as MinGEQ-Multi-CDGP-IP, is obtained by making two modifications to the previous model. The first one is changing the RHS of constraints (3.12) from 1 to $q_{i}$, which ensures that, instead of receiving only one color, each vertex $i$ will receive $q_{i}$ colors. The second one is expanding the set of constraints (3.13) in order to add new ones for ensuring that the same vertex $i$ avoids using colors that violate the distance $d_{i, i}$, that is, there will be one constraint for each $(i, j) \in E ; 1 \leq c, e \leq U$ such that $|c-e|<d_{i, j}$ and also for each $i \in V ; 1 \leq c, e \leq U$ such that $|c-e|<d_{i, i}$. Note that this is equivalent to having an edge $(i, i)$ for each vertex $i$, which would make the new constraints be automatically included in the original constraint set. The full MinGEQ-Multi-CDGP-IP formulation we propose is given below. These modifications do not impact the asymptotic size of the formulation.

$$
\begin{array}{cll}
\text { Minimize } & z_{\max } & \\
\text { Subject to } & \sum_{c=1}^{U} x_{c i}=q_{i} & (\forall i \in V) \\
& x_{i c}+x_{j e} \leq 1 & \left(\forall(i, j) \in E ; 1 \leq c, e \leq U|| c-e \mid<d_{i, j}\right) \\
x_{i c}+x_{i e} \leq 1 & \left(\forall i \in V ; 1 \leq c, e \leq U|| c-e \mid<d_{i, i}\right) \\
z_{\max } \geq c x_{i c} & (\forall i \in V ; 1 \leq c \leq U) \\
x_{c i} \in\{0,1\} & (\forall i \in V ; 1 \leq c \leq U) \\
& z_{\max } \in \mathbb{R} &
\end{array}
$$

Another formulation that can be used to solve MinGEQ-Multi-CDGP (and MinGEQ-CDGP) is based on the one by [11], which was originally proposed for channel assignment problems. Instead of using a single integer variable to track the coloring span ( $z_{\max }$ in the above IP formulations), a set of binary variables are used instead, as defined below:

$-u_{c}= \begin{cases}1 & \text { if color } c \text { is the maximum one used in the solution; } \\ 0 & \text { otherwise. }\end{cases}$ 
TABLE 1. Summary of constraint and integer programming formulations.

\begin{tabular}{|c|c|c|c|c|}
\hline Formulation & Type & Problem & \# Vars & \# Constr. \\
\hline MinGEQ-CDGP-CP & & $\mathrm{BCP}$ & $O(|V|)$ & $O(|E|)$ \\
\hline MinGEQ-CDGP-Unif-CP & $\begin{array}{l}\text { Constraint } \\
\text { programming }\end{array}$ & $\begin{array}{l}\text { BCP w/ } \\
\text { Unif. Dist. } \\
\text { (Complete } \\
\text { graphs) }\end{array}$ & $O(|V|)$ & $O(|V|)$ \\
\hline MinGEQ-Multi-CDGP-CP & & $\mathrm{BMCP}$ & $O\left(|V| q_{\max }\right)$ & $O\left(|E| q_{\max }\right)$ \\
\hline MinGEQ-CDGP-IP & & $\mathrm{BCP}$ & $O(U|V|)$ & $O\left(U|V|+U^{2}|E|\right)$ \\
\hline MinGEQ-Multi-CDGP-IP & $\begin{array}{l}\text { Integer } \\
\text { programming }\end{array}$ & $\mathrm{BMCP}$ & $O(U|V|)$ & $O\left(U|V|+U^{2}|E|\right)$ \\
\hline $\begin{array}{l}\text { MinGEQ-Multi-CDGP-IP- } \\
\text { FullBinary }\end{array}$ & & $\mathrm{BMCP}$ & $O((U|V|)+U)$ & $O\left(U^{2}|V|+U|V|+U^{2}|E|\right)$ \\
\hline
\end{tabular}

The resulting model, which we will denote as MinGEQ-Multi-CDGP-IP-FullBinary, is then given by:

$$
\begin{array}{cll}
\text { Minimize } & \sum_{c=1}^{U} c u_{c} & \\
\text { Subject to } & \sum_{c=1}^{U} x_{c i}=q_{i} & (\forall i \in V) \\
& x_{i c}+x_{j e} \leq 1 & \left(\forall(i, j) \in E ; 1 \leq c, e \leq U|| c-e \mid<d_{i, j}\right) \\
x_{i c}+x_{i e} \leq 1 & \left(\forall i \in V ; 1 \leq c, e \leq U|| c-e \mid<d_{i, i}\right) \\
\sum_{c=1}^{U} u_{c}=1 & \\
x_{i c}+u_{e} \leq 1 & \\
x_{c i} \in\{0,1\} & (\forall i \in V ; 1 \leq c, e \leq U \mid c>e) \\
u_{c} \in\{0,1\} & (\forall i \in V ; 1 \leq c \leq U) \\
& (\forall 1 \leq c \leq U)
\end{array}
$$

In MinGEQ-Multi-CDGP-IP-FullBinary, constraint sets (3.25), (3.26) and (3.27) are the same as sets (3.18), (3.19) and (3.20) from MinGEQ-Multi-CDGP-IP. Constraints (3.28) ensure that only one color can be the maximum one used. The set of constraints (3.29) require that, if a certain color is the maximum one used, then no vertex can receive a color higher than the maximum. This formulation has $O((U|V|)+U)$ variables and $O\left(U^{2}|V|+U|V|+U^{2}|E|\right)$ constraints, making it larger than MinGEQ-Multi-CDGP-IP.

A summary of the constraint and integer programming formulations described in this section is given in Table 1.

\subsection{Upper bounds for color sets}

Both CP and IP models need a finite color set, which, as shown previously, consist of an interval of integers $[1, \ldots, U]$, where $U$ is an upper bound for the coloring span. A trivial value for $U$ can be calculated by summing the distances imposed on all edges plus 1 , that is, $U=\sum_{(i, j) \in E} d_{i, j}+1$. However, this upper bound is very weak, since, by summing all distances, we are ignoring the fact that colors can be reused by vertices not adjacent to each other, which makes the coloring span become a large value far from optimum. This also makes the color set have a large cardinality, which has a huge impact on the computing performance of these models, especially 
the IP model, since the number of variables and constraints are proportional to the upper bound, which can lead to out-of-memory scenarios.

A better value for $U$ is given by preprocessing the input graph, where a heuristic which does not need an explicit upper bound is applied to it. The span of the resulting solution is used as the value $U$ when assembling CP and IP models for the input graph. A greedy algorithm for coloring the input graph can be used for this, where the vertices are processed following an order based on their color demands - vertices with higher demands are colored first. A color for a vertice $i$ is determined by first setting its color candidate as num $\operatorname{Col}[i] \times d_{i, i}+1$, where $n u m C o l[i]$ is the number of colors already assigned to $i$, and checking if it violates any separation constraint with any of its neighbor vertices. If a violation occurs, the color candidate is incremented by 1 and this checking is made again until a feasible color is found. The color is then assigned to $i$ and, if its demands are not fully satisfied, an additional color is calculated and assigned to it. This is repeated until the graph is fully colored. Algorithm 1 gives pseudocode for this greedy heuristic. The upper bound is, then, the span of the solution returned by this method.

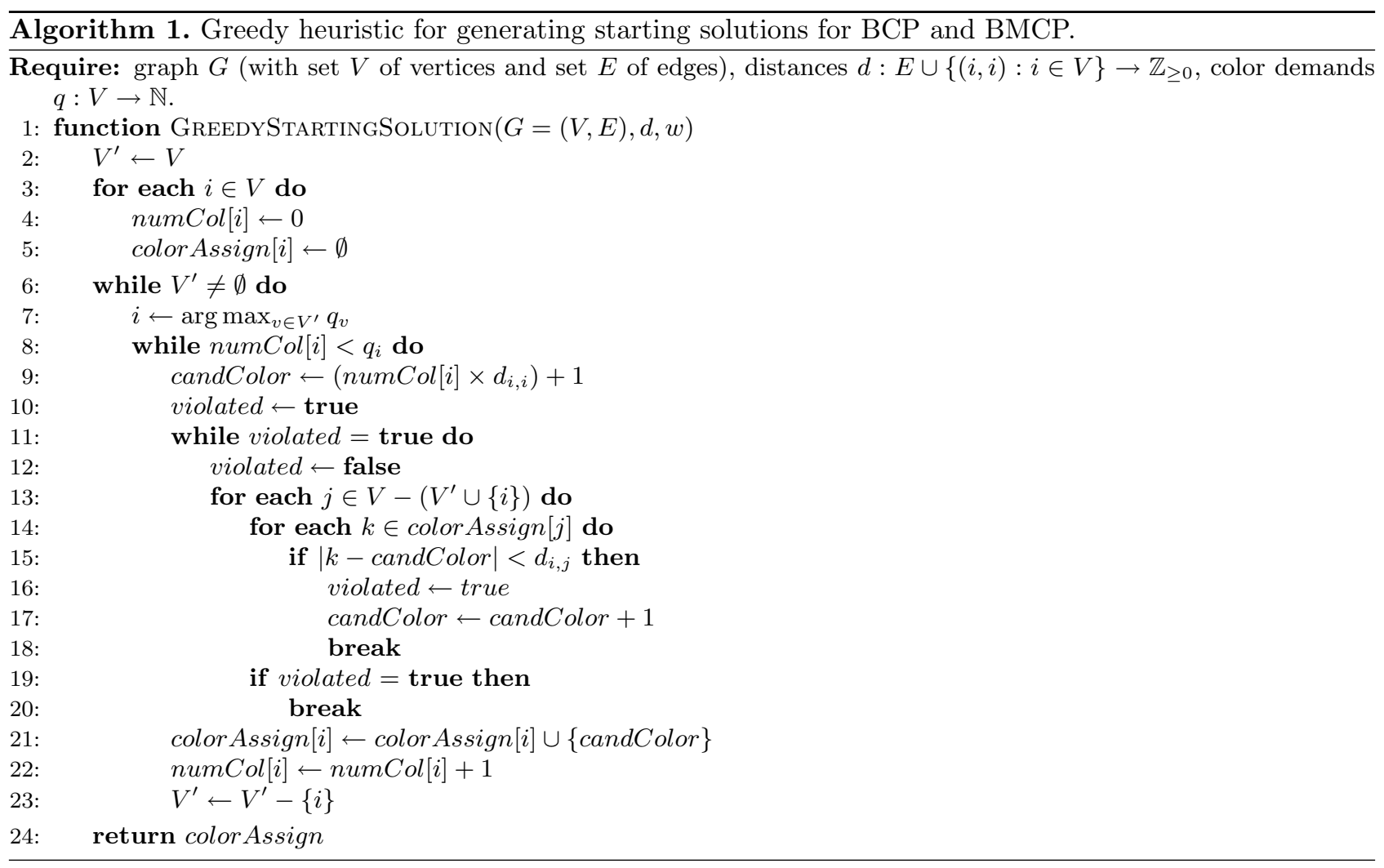

\section{Computational experiments}

The constraint and integer programming formulations were implemented in $\mathrm{C}++$ using IBM/ILOG CPLEX solver, version 12.5.1, and its CP Optimizer component. The resulting programs were executed in a Microsoft Azure A9 Virtual Machine, consisting of Intel Xeon E5-2670 processors (16 cores @ 2.6 GHz), $112 \mathrm{~GB}$ of RAM and Ubuntu Linux 14.04.1 LTS operating system. Both formulations used the standard parameters of the solver, but using only one thread, and each instance was limited to 48 hours of CPU time (172 $800 \mathrm{~s})$.

We used two benchmarks from the literature in our experiments. The first set of literature instances is known as GEOM, generated by Michael Trick [26] for BCP and its multicoloring variant, BMCP, and consists of 33 


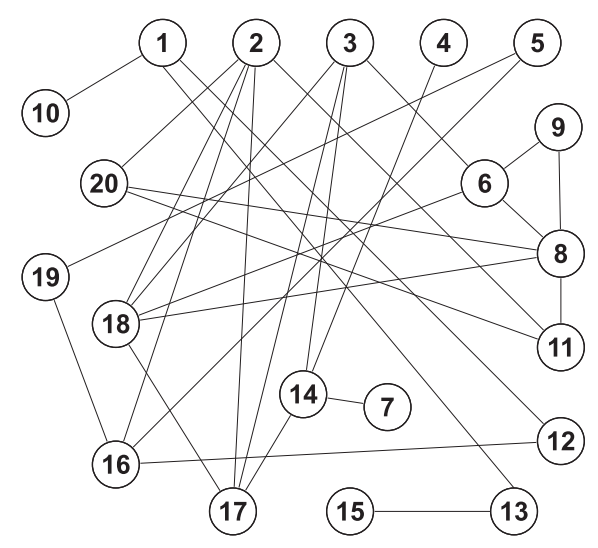

Figure 5. Graph layout of instance GEOM20b.

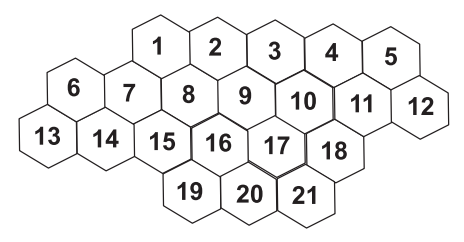

(a) Philadelphia.

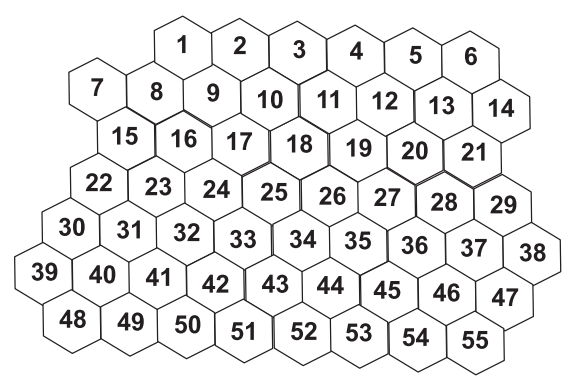

(b) Artificial.

Figure 6. Cellular networks used in Philadelphia and Artificial (55-cell) instances.

instances of three types: GEOM $n$ are sparse graphs, while GEOM $n$ a and GEOMnb are denser graphs (where $n$ is the number of vertices in the instance). An example of graph from this set of instances is given in Figure 5, where the graph from instance GEOM20b is shown.

The second set of instances consists of the classic Philadelphia (21 stations) and Helsinki (25 vertices) problems for MS-CAP, based on cellular networks from the cities of the same names, and an artificial problem (55 vertices) that extends a Philadelphia instance, as seen in $[2,6,15]$. The cellular models (hexagonal cells) for Philadelphia and artificial instances are given in Figure 6.

We remark that, for MS-CAP instances, the graph induced by the cellular network is not directly used as $\mathrm{BCP} / \mathrm{BMCP}$ instances. Rather, another one is determined from the network layout, called interference graph, which takes into account the minimum distance between each pair of cells. The instances defined on such networks are based on a two-band buffering system, that is, each cell interferes only with others situated at most 2 units of distance apart [1]. To exemplify this concept, the interference graph for the 21-cell Philadelphia network is given in Figure 7. Since these instances are defined for BMCP only, we applied the suggestion by the authors of the GEOM instances in these as well to generate BCP instances.

When constructing the models for each instance, we executed the preprocessing discussed in Section 3.1 in order to obtain a feasible solution and an upper bound. To further help the solvers, we fed the entire starting solution to them, namely, we passed the solution as a starting point to CP Optimizer and as a MIP start to CPLEX, instead of only using the span as an upper bound. This is especially important for CPLEX, since it guarantees that an optimality gap is calculated as soon as the enumeration starts. 


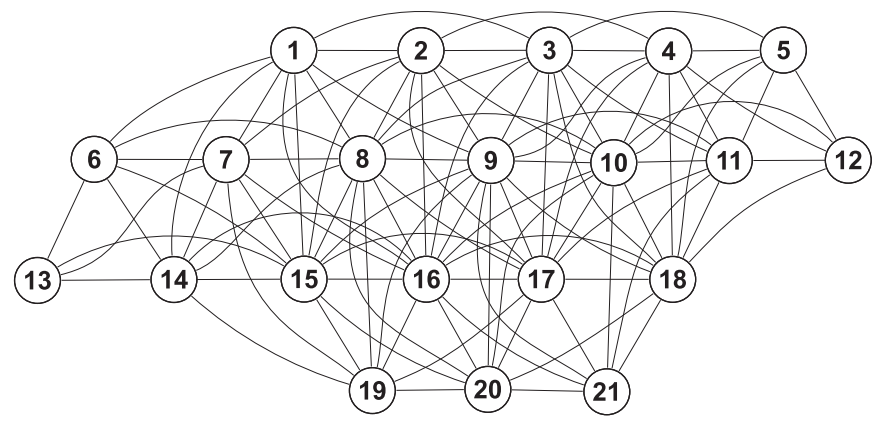

FiguRE 7. Interference graph for the 21-cell Philadelphia network. The distances imposed on the edges, as well as multicoloring demands, which would define loop edges, were omitted in the graph.
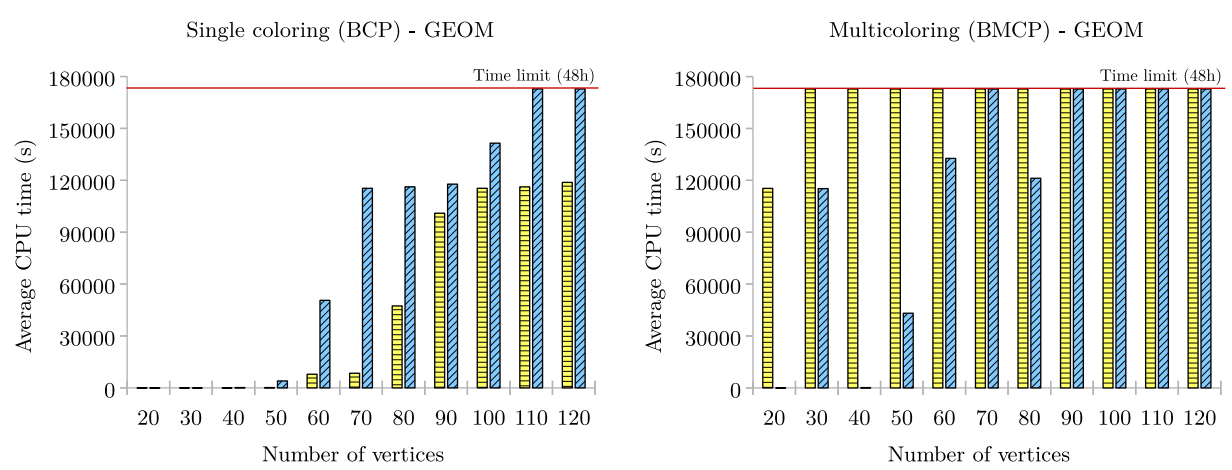

目 Constraint Programming $\boxminus$ Integer Programming

FIgURE 8 . Number of vertices $\times$ CPU time needed to prove optimality (if found) for each method on GEOM instances.

The first results presented are for BCP. Table 2 shows the results for the GEOM BCP instances, where underlined values for the span (in Best Found and Best Reported columns) indicate that it is proved to be optimal using at least one of the exact approaches (CP or IP). Since the BCP variants are also used in the literature, we compared our results with the Discropt heuristic framework in [24] and the multistart iterated tabu search heuristic in [18] to verify the correctness of the solutions by the CP and IP formulations. For all sparse instances (the ones without "a" or "b" in the name), the constraint programming implementation was able to prove optimality. However, we emphasize that, for some instances, no method achieved the best solution presented in [24]. As noted in [18], no other work has obtained the same results, while our exact approaches reached the same best solutions for these instances obtained by other authors, which leads us to believe there is a mistake in [24], as marked in Table 2.

Table 3 shows the results for MS-CAP (Philadelphia, Helsinki and Artificial) instances without considering multicoloring demands. We note that, for each Philadelphia constraint matrix $C_{21}^{i}$ where $i$ is odd, by dropping the multicoloring demands, the instances become equal, since the only difference among them is the separation between colors of the same vertex. The same occurs for each even $i$. Given that, we grouped together results for each odd $i(1,3,5$ and 7$)$ and for each even $i(2,4,6,8)$. Again, the CP formulation reaches optimality for each instance much faster, although runtimes are small due to the size of these relaxed instances. 
TABLE 2. Results for the constraint and integer programming formulations applied on literature BCP instances (GEOM set). Underlined results in "Best Found" columns are optimal values.

\begin{tabular}{|c|c|c|c|c|c|c|c|c|c|c|}
\hline \multirow{2}{*}{ Instance } & \multirow[t]{2}{*}{$|V|$} & \multirow[t]{2}{*}{$|E|$} & \multirow{2}{*}{$\begin{array}{l}\text { Phan } \\
\text { and } \\
\text { Skiena } \\
{[24]} \\
\text { Best } \\
\text { reported }\end{array}$} & \multirow{2}{*}{$\begin{array}{l}\text { Lai and } \\
\text { Lu [18] } \\
\\
\text { Best } \\
\text { reported }\end{array}$} & \multicolumn{2}{|c|}{$\begin{array}{c}\text { Constr. Progr. (CP } \\
\text { Optimizer) }\end{array}$} & \multicolumn{4}{|c|}{ Integer Progr. (CPLEX) } \\
\hline & & & & & $\begin{array}{l}\text { Best } \\
\text { found }\end{array}$ & Time (s) & Best found & Best LB & Gap (\%) & Time (s) \\
\hline GEOM20 & & 40 & $20^{*}$ & 21 & 21 & 0.00 & $\underline{21}$ & 21.000 & 0.00 & 0.33 \\
\hline GEOM20a & 20 & 57 & 20 & 20 & $\underline{\overline{20}}$ & 0.02 & $\underline{\overline{20}}$ & 20.000 & 0.00 & 0.95 \\
\hline GEOM20b & & 52 & 13 & 13 & $\overline{13}$ & 0.01 & $\overline{13}$ & 13.000 & 0.00 & 0.09 \\
\hline GEOM30 & & 80 & $27^{*}$ & 28 & 28 & 0.05 & 28 & 28.000 & 0.00 & 0.88 \\
\hline GEOM30a & 30 & 111 & 27 & 27 & 27 & 0.05 & 27 & 27.000 & 0.00 & 8.06 \\
\hline GEOM $30 b$ & & 111 & 26 & 26 & 26 & 0.03 & 26 & 26.000 & 0.00 & 2.27 \\
\hline GEOM40 & & 118 & $27^{*}$ & 28 & 28 & 0.05 & 28 & 28.000 & 0.00 & 1.97 \\
\hline GEOM40a & 40 & 186 & 38 & 37 & 37 & 1.39 & $\frac{37}{37}$ & 37.000 & 0.00 & 278.66 \\
\hline GEOM40b & & 197 & 36 & 33 & $\underline{33}$ & 2.06 & $\underline{33}$ & 33.000 & 0.00 & 252.39 \\
\hline GEOM50 & & 177 & 29 & 28 & $\underline{28}$ & 0.26 & $\underline{28}$ & 28.000 & 0.00 & 21.44 \\
\hline GEOM50a & 50 & 288 & 54 & 50 & $\underline{50}$ & 374.42 & $\underline{50}$ & 50.000 & 0.00 & 3457.25 \\
\hline GEOM50b & & 299 & 40 & 35 & $\underline{35}$ & 144.69 & $\underline{35}$ & 35.000 & 0.00 & 8494.52 \\
\hline GEOM60 & & 245 & 34 & 33 & $\underline{\overline{33}}$ & 1.12 & $\underline{\overline{33}}$ & 33.000 & 0.00 & 45.73 \\
\hline GEOM60a & 60 & 339 & 54 & 50 & $\underline{50}$ & 684.59 & $\underline{50}$ & 50.000 & 0.00 & 16755.65 \\
\hline GEOM60b & & 426 & 47 & 41 & $\underline{\overline{41}}$ & 22915.94 & $\underline{\overline{41}}$ & 41.000 & 0.00 & 134996.77 \\
\hline GEOM70 & & 337 & 40 & 38 & $\underline{38}$ & 2.39 & $\underline{38}$ & 38.000 & 0.00 & 533.53 \\
\hline GEOM70a & 70 & 529 & 64 & 61 & $\underline{61}$ & 24798.03 & $\leq 62$ & 38.000 & 38.71 & 172815.55 \\
\hline GEOM70b & & 558 & 54 & 47 & $\underline{47}$ & 534.65 & $\leq 49$ & 44.0000 & 10.20 & 172834.40 \\
\hline GEOM80 & & 429 & 44 & 41 & $\underline{41}$ & 8.18 & $\underline{41}$ & 41.000 & 0.00 & 3019.18 \\
\hline GEOM80a & 80 & 692 & 69 & 63 & $\underline{63}$ & 87770.77 & $\leq 65$ & 39.0000 & 40.00 & 172803.55 \\
\hline GEOM $80 b$ & & 743 & 70 & 60 & $\underline{60}$ & 54320.89 & $\leq 66$ & 21.0000 & 68.18 & 172800.25 \\
\hline GEOM90 & & 531 & 48 & 46 & $\underline{46}$ & 55.18 & 46 & 46.000 & 0.00 & 7768.62 \\
\hline GEOM90a & 90 & 879 & 74 & 63 & $\underline{63}$ & 130050.12 & $\leq 72$ & 7.000 & 90.28 & 173100.57 \\
\hline GEOM90b & & 950 & 83 & 69 & $\overline{\leq 69}$ & 172800.00 & $\leq 85$ & 2.1127 & 97.51 & 172802.83 \\
\hline GEOM100 & & 647 & 55 & 50 & $\underline{50}$ & 545.79 & $\underline{50}$ & 50.0000 & 0.00 & 78836.94 \\
\hline GEOM100a & 100 & 1092 & 84 & 67 & $\leq 70$ & 172800.01 & $\leq 85$ & 3.0863 & 96.37 & 172824.54 \\
\hline GEOM100b & & 1150 & 87 & 72 & $\leq 71$ & 172800.02 & $\leq 101$ & 2.2271 & 97.75 & 172840.38 \\
\hline GEOM110 & & 748 & 59 & 50 & 50 & 2982.24 & $\underline{50}$ & 50.0000 & 0.00 & 170043.88 \\
\hline GEOM110a & 110 & 1317 & 88 & 72 & $\overline{\leq 73}$ & 172800.01 & $\overline{\leq 97}$ & 2.1963 & 97.70 & 172811.66 \\
\hline GEOM110b & & 1366 & 87 & 78 & $\leq 79$ & 172800.01 & $\leq 99$ & 2.2208 & 97.76 & 172821.35 \\
\hline GEOM120 & & 893 & 67 & 59 & 59 & 10778.18 & $\leq 60$ & 24.0000 & 60.00 & 173296.11 \\
\hline GEOM120a & 120 & 1554 & 101 & 82 & $\leq 84$ & 172800.01 & $\leq 110^{\dagger}$ & 2.1039 & 98.09 & 173181.91 \\
\hline GEOM120b & & 1611 & 103 & 84 & $\leq 85$ & 172800.01 & $\leq 113^{\dagger}$ & 2.1245 & 98.12 & 173187.16 \\
\hline
\end{tabular}

${ }^{*}$ Results lower than the obtained optimum - possibly wrong in the corresponding work. ${ }^{\dagger}$ No solution found by B\&C, but CPLEX returns the initial greedy heuristic solution.

The next experiments are for BMCP. For these instances, a trivial lower bound can be calculated as $L=\max \left[\left(d_{i, i}\left(q_{i}-1\right)\right)+1\right]$, as shown by [2]. This value is also inserted in the models by adding a single constraint requiring that the objective value be greater than or equal to $L$, which helps the enumeration end faster when the optimal solution has span equivalent to this lower bound, especially when using CP. It also enables IP to report an optimality gap even when the root node takes too much time to find even the linear relaxation solution.

Table 4 shows results obtained for the GEOM instances. We compared our results for these instances with the same multistart iterated tabu search heuristic from [18], where the algorithm for BCP is applied to BMCP instances by expanding the vertices into cliques as shown in Section 2. Furthermore, Table 5 shows results 
TABLE 3. Results for the constraint and integer programming formulations applied on literature MS-CAP instances (Philadelphia, Helsinki and Artificial) without multicoloring demands. Underlined results in "Best Found" columns are optimal values. Since IP reaches all optimal solutions, the best LB has been omitted in the table.

\begin{tabular}{|c|c|c|c|c|c|c|}
\hline \multirow{2}{*}{$\begin{array}{l}\text { Const. } \\
\text { matrix }\end{array}$} & \multirow{2}{*}{$|V|$} & \multirow{2}{*}{$|E|$} & \multicolumn{2}{|c|}{ Constr. Progr. (CP optimizer) } & \multicolumn{2}{|c|}{ Integer Progr. (CPLEX - B\&C) } \\
\hline & & & Best found & Time (s) & Best found & Time (s) \\
\hline $\begin{array}{l}C_{21}^{1}, C_{21}^{3}, C_{21}^{5} \\
\text { and } C_{21}^{7}\end{array}$ & 21 & 102 & $\underline{7}$ & 0.40 & $\underline{7}$ & 0.87 \\
\hline $\begin{array}{l}C_{21}^{2}, C_{21}^{4}, C_{21}^{6} \\
\text { and } C_{21}^{8}\end{array}$ & & & $\underline{9}$ & 0.06 & $\underline{9}$ & 2.66 \\
\hline$C_{25}^{1}$ & 25 & 134 & $\underline{8}$ & 4.71 & $\underline{8}$ & 1.90 \\
\hline$C_{55}^{\perp}$ & 55 & 362 & 7 & 0.79 & 7 & 30.63 \\
\hline
\end{tabular}

TABLE 4. Results for the constraint and integer programming formulations applied on literature BMCP instances (GEOM set). Underlined results in "Best Found" columns are optimal values.

\begin{tabular}{|c|c|c|c|c|c|c|c|c|c|c|}
\hline \multirow[b]{2}{*}{ Instance } & \multirow[b]{2}{*}{$|V|$} & \multirow[b]{2}{*}{$|E|$} & \multirow[b]{2}{*}{ Trivial LB } & \multirow{2}{*}{$\frac{\text { Lai and } \mathrm{Lu}[18]}{\text { Best reported }}$} & \multicolumn{2}{|c|}{ Constr. Progr. (CP Optimizer) } & \multicolumn{4}{|c|}{ Integer Progr. (CPLEX - B\&C) } \\
\hline & & & & & Best found & Time $(\mathrm{s})$ & Best found & Best LB & Gap (\%) & Time (s) \\
\hline GEOM20 & & 40 & 91 & 149 & $\leq 149$ & 172800.01 & $\underline{149}$ & 149.0000 & 0.00 & 15.17 \\
\hline GEOM20a & 20 & 57 & 91 & 169 & $\leq 169$ & 172800.01 & $\underline{169}$ & 169.0000 & 0.00 & 18.49 \\
\hline GEOM20b & & 52 & 21 & 44 & $\underline{44}$ & 476.92 & $\underline{44}$ & 44.0000 & 0.00 & 1.58 \\
\hline GEOM30 & & 80 & 91 & 160 & $\leq 160$ & 172800.01 & $\leq 160$ & 159.0000 & 0.62 & 172830.72 \\
\hline GEOM30a & 30 & 111 & 91 & 209 & $\leq 215$ & 172800.01 & $\leq 211$ & 189.0000 & 10.43 & 172813.47 \\
\hline GEOM30b & & 111 & 21 & 77 & $\leq 77$ & 172800.00 & $\underline{77}$ & 77.0000 & 0.00 & 41.87 \\
\hline GEOM40 & & 118 & 91 & 167 & $\leq 168$ & 172800.01 & $\underline{167}$ & 167.0000 & 0.00 & 1192.28 \\
\hline GEOM40a & 40 & 186 & 91 & 213 & $\leq 225$ & 172800.01 & $\overline{213}$ & 213.0000 & 0.00 & 111262.08 \\
\hline GEOM40b & & 197 & 21 & 74 & $\leq 74$ & 172800.00 & $\underline{74}$ & 74.0000 & 0.00 & 17027.77 \\
\hline GEOM50 & & 177 & 91 & 224 & $\leq 226$ & 172800.02 & $\underline{224}$ & 224.0000 & 0.00 & 52450.85 \\
\hline GEOM50a & 50 & 288 & 91 & 314 & $\leq 332$ & 172800.03 & $\overline{\leq 361}$ & 95.5218 & 73.54 & 172820.13 \\
\hline GEOM50b & & 299 & 21 & 83 & $\leq 85$ & 172800.00 & $\leq 87$ & 52.0000 & 40.23 & 172819.47 \\
\hline GEOM60 & & 245 & 91 & 258 & $\leq 259$ & 172800.02 & 258 & 258.0000 & 0.00 & 156987.80 \\
\hline GEOM60a & 60 & 339 & 91 & 356 & $\overline{\leq} 380$ & 172800.03 & $\overline{\leq 448}$ & 93.5801 & 78.93 & 172813.01 \\
\hline GEOM60b & & 426 & 21 & 113 & $\leq 117$ & 172800.01 & $\leq 125$ & 34.0000 & 72.80 & 172897.07 \\
\hline GEOM70 & & 337 & 91 & 270 & $\leq 284$ & 172800.03 & $\leq 305$ & 94.2092 & 69.11 & 172804.56 \\
\hline GEOM70a & 70 & 529 & 91 & 467 & $\leq 483$ & 172800.05 & $\leq 578$ & 91.0000 & 84.26 & 172839.51 \\
\hline GEOM70b & & 558 & 21 & 116 & $\leq 123$ & 172800.01 & $\leq 134$ & 22.7359 & 83.03 & 172805.88 \\
\hline GEOM80 & & 429 & 91 & 381 & $\leq 395$ & 172800.04 & $\leq 511$ & 95.2644 & 80.19 & 172809.70 \\
\hline GEOM80a & 80 & 692 & 91 & 361 & $\leq 382$ & 172800.05 & $\leq 479$ & 91.0000 & 81.00 & 172885.02 \\
\hline GEOM80b & & 743 & 21 & 139 & $\leq 145$ & 172800.01 & $\leq 170$ & 23.0547 & 86.44 & 172820.56 \\
\hline GEOM90 & & 531 & 91 & 330 & $\leq 342$ & 172800.05 & $\leq 423$ & 93.2736 & 77.73 & 172811.82 \\
\hline GEOM90a & 90 & 879 & 91 & 375 & $\leq 392$ & 172800.07 & $\leq 452$ & 91.0000 & 79.87 & 172830.60 \\
\hline GEOM90b & & 950 & 21 & 144 & $\leq 156$ & 172800.01 & $\leq 212$ & 22.2574 & 89.50 & 172844.07 \\
\hline GEOM100 & & 647 & 91 & 404 & $\leq 426$ & 172800.07 & $\leq 493$ & 94.2797 & 80.88 & 173190.69 \\
\hline GEOM100a & 100 & 1092 & 91 & 442 & $\leq 465$ & 172800.08 & $\leq 596$ & 91.0000 & 84.73 & 172871.84 \\
\hline GEOM100b & & 1150 & 21 & 156 & $\leq 169$ & 172800.02 & $\leq 220$ & 21.0000 & 90.45 & 172810.33 \\
\hline GEOM110 & & 748 & 91 & 381 & $\leq 399$ & 172800.07 & $\leq 500$ & 91.0000 & 81.80 & 172840.98 \\
\hline GEOM110a & 110 & 1317 & 91 & 488 & $\leq 527$ & 172800.10 & $\leq 610$ & 91.0000 & 85.08 & 173095.42 \\
\hline GEOM110b & & 1366 & 21 & 204 & $\leq 207$ & 172800.01 & $\leq 250$ & 22.0001 & 91.20 & 172835.82 \\
\hline GEOM120 & & 893 & 91 & 396 & $\leq 427$ & 172800.06 & $\leq 505$ & 93.9762 & 81.39 & 172923.18 \\
\hline GEOM120a & 120 & 1554 & 91 & 554 & $\leq 585$ & 172800.16 & $\leq 641$ & 91.0000 & 85.80 & 173312.14 \\
\hline GEOM120b & & 1611 & 21 & 189 & $\leq 202$ & 172800.01 & $\leq 247$ & 21.8723 & 91.14 & 172852.82 \\
\hline
\end{tabular}


TABLE 5. Results for the constraint and integer programming formulations applied on literature MS-CAP instances (Philadelphia, Helsinki and Artificial). Underlined results in "Best Found" columns are optimal values. Since IP reaches all optimal solutions, the best LB has been omitted in the table.

\begin{tabular}{|c|c|c|c|c|c|c|c|c|c|c|c|}
\hline \multirow[t]{2}{*}{$\begin{array}{l}\text { Const. } \\
\text { matr. }\end{array}$} & \multirow[t]{2}{*}{$\begin{array}{l}\text { Demd. } \\
\text { vect. }\end{array}$} & \multirow[t]{2}{*}{$|V|$} & \multirow[t]{2}{*}{$|E|$} & \multirow{2}{*}{$\begin{array}{l}\text { Lower } \\
\text { bound }\end{array}$} & \multicolumn{2}{|c|}{$\begin{array}{l}\text { Chakraborty Kendall } \\
{[2] \quad \text { and }} \\
\text { Mohamad } \\
{[15]}\end{array}$} & $\begin{array}{l}\text { Kim } \\
\text { et al. [16] }\end{array}$ & \multicolumn{2}{|c|}{$\begin{array}{l}\text { Constr. Progr. } \\
\text { (CP Optimizer) }\end{array}$} & \multicolumn{2}{|c|}{$\begin{array}{l}\text { Integer Progr. } \\
(\text { CPLEX - B\&C) }\end{array}$} \\
\hline & & & & & $\begin{array}{l}\text { Best } \\
\text { reported }\end{array}$ & $\begin{array}{l}\text { Best } \\
\text { reported }\end{array}$ & $\begin{array}{l}\text { Best } \\
\text { reported }\end{array}$ & Best found & Time (s) & Best found & Time (s) \\
\hline$C_{21}^{1}$ & $D_{21}^{1}$ & \multirow{16}{*}{21} & \multirow{16}{*}{102} & 533 & 533 & 533 & 533 & $\underline{533}$ & 4.20 & $\underline{533}$ & 0.50 \\
\hline$C_{21}^{1}$ & $D_{21}^{2}$ & & & 309 & 309 & 309 & 309 & $\underline{309}$ & 1.34 & $\underline{309}$ & 1.22 \\
\hline$C_{21}^{2}$ & $D_{21}^{1}$ & & & 533 & 533 & 533 & 533 & $\underline{533}$ & 10.53 & $\underline{533}$ & 308.04 \\
\hline$C_{21}^{2}$ & $D_{21}^{2}$ & & & 309 & 309 & 309 & 309 & $\underline{309}$ & 625.93 & $\underline{309}$ & 165.54 \\
\hline$C_{21}^{3}$ & $D_{21}^{1}$ & & & 457 & 457 & 457 & - & $\underline{457}$ & 3.96 & $\underline{457}$ & 0.39 \\
\hline$C_{21}^{3}$ & $D_{21}^{2}$ & & & 265 & 265 & 265 & - & $\underline{265}$ & 3.54 & $\underline{265}$ & 1.52 \\
\hline$C_{21}^{4}$ & $D_{21}^{1}$ & & & 457 & 457 & 457 & - & $\overline{457}$ & 41.24 & $\underline{457}$ & 202.01 \\
\hline$C_{21}^{4}$ & $D_{21}^{2}$ & & & 265 & 265 & 265 & - & $\leq 266$ & 172800.06 & $\underline{265}$ & 214.01 \\
\hline$C_{21}^{5}$ & $D_{21}^{1}$ & & & 381 & 381 & 381 & 381 & $\underline{381}$ & 3.23 & $\underline{381}$ & 0.29 \\
\hline$C_{21}^{5}$ & $D_{21}^{2}$ & & & 221 & 221 & 221 & 221 & $\underline{221}$ & 100.81 & $\underline{221}$ & 5.09 \\
\hline$C_{21}^{6}$ & $D_{21}^{1}$ & & & 381 & 463 & 435 & 427 & $\leq 449$ & 172800.08 & $\underline{427}$ & 6827.49 \\
\hline$C_{21}^{6}$ & $D_{21}^{2}$ & & & 221 & 273 & 268 & 253 & $\leq 266$ & 172800.04 & $\underline{253}$ & 2026.67 \\
\hline$C_{21}^{7}$ & $D_{21}^{1}$ & & & 305 & 305 & 305 & - & $\underline{305}$ & 12.85 & $\underline{305}$ & 1.10 \\
\hline$C_{21}^{7}$ & $D_{21}^{2}$ & & & 177 & 197 & 185 & - & $\leq 180$ & 172800.06 & $\underline{180}$ & 24.54 \\
\hline$C_{21}^{8}$ & $D_{21}^{1}$ & & & 305 & 465 & 444 & - & $\leq 435$ & 172800.07 & $\underline{427}$ & 1185.27 \\
\hline$C_{21}^{8}$ & $D_{21}^{2}$ & & & 177 & 278 & 271 & - & $\leq 267$ & 172800.06 & $\underline{253}$ & 1116.45 \\
\hline$C_{25}^{1}$ & $D_{25}^{3}$ & \multirow{2}{*}{25} & \multirow{2}{*}{134} & 21 & 73 & 73 & - & $\leq 73$ & 172800.00 & $\underline{73}$ & 1.10 \\
\hline$C_{25}^{1}$ & $D_{25}^{4}$ & & & 89 & $121^{*}$ & 200 & - & $\leq 200$ & 172800.07 & $\underline{200}$ & 2.18 \\
\hline$C_{55}^{1}$ & $D_{55}^{5}$ & \multirow{2}{*}{55} & \multirow{2}{*}{362} & 309 & 309 & 309 & - & $\underline{309}$ & 11078.95 & $\underline{309}$ & 460.12 \\
\hline$C_{55}^{1}$ & $D_{55}^{6}$ & & & 71 & 79 & 72 & - & $\underline{71}$ & 6.33 & $\underline{71}$ & 28.56 \\
\hline
\end{tabular}

${ }^{*}$ Results lower than the obtained optimum - possibly wrong in the corresponding work.

for the MS-CAP (Philadelphia, Helsinki and Artificial) instances in their original forms (that is, including multicoloring), which were compared with the constructive heuristic from [2], the local search algorithm from [15] and the memetic algorithm from [16].

For BMCP, the efficiency between CP and IP is practically reversed: most instances are solved to optimality faster with IP than CP. In fact, for the MS-CAP instances, we were able to obtain all optimal values using IP. This is explained by taking into account, as discussed in Section 3, that the IP model is able to consider multicoloring demands without expanding the number of vertices, only having to change a set of constraints and add another set for the different colors for each vertex. However, the CP model has to grow more, since, essentially, a BMCP instance is treated as a special BCP one, since the number of variables increases and a new set of constraints is introduced. Figure 8 shows this difference in efficiency between methods. However, CP still has an advantage in BMCP: when it is unable to solve a problem to optimality in the given time limit, the solution that it returns has a better quality than the one found by IP (that is, the coloring span of the solution found by $\mathrm{CP}$ is lower than the one found by IP). This happens because CP has explicit information about which colors each vertex can assume, instead of calculating such colors. 
TABLE 6. Number of branches and fails (for CP) and of global cuts of each type (for IP) applied to GEOM instances with unit demands.

\begin{tabular}{|c|c|c|c|c|c|c|c|c|}
\hline \multirow{2}{*}{ Instance } & \multirow{2}{*}{$\begin{array}{l}\text { Num. } \\
\text { Vert. }\end{array}$} & \multicolumn{2}{|c|}{ Const. Prog. (CP Optimizer) } & \multicolumn{5}{|c|}{ Integer Progr. (CPLEX) } \\
\hline & & \# Branches & \# Fails & $\begin{array}{l}\# \\
\text { Clique } \\
\text { cuts }\end{array}$ & $\begin{array}{l}\# \\
\text { Implied } \\
\text { bound } \\
\text { cuts }\end{array}$ & $\begin{array}{l}\text { \# MIP } \\
\text { rounding } \\
\text { cuts }\end{array}$ & $\begin{array}{l}\# \\
\text { Zero-half } \\
\text { cuts }\end{array}$ & $\begin{array}{l}\text { \# Gomory } \\
\text { fractional } \\
\text { cuts }\end{array}$ \\
\hline GEOM20 & & 972 & 462 & 9 & 15 & 0 & 8 & 10 \\
\hline GEOM20a & 20 & 4667 & 2298 & 3 & 27 & 0 & 2 & 9 \\
\hline GEOM20b & & 2224 & 1096 & 14 & 7 & 0 & 5 & 0 \\
\hline GEOM30 & & 10102 & 4782 & 13 & 62 & 0 & 4 & 31 \\
\hline GEOM30a & 30 & 9024 & 4353 & 25 & 473 & 0 & 20 & 60 \\
\hline GEOM30b & & 6651 & 3210 & 9 & 194 & 0 & 11 & 49 \\
\hline GEOM40 & & 9965 & 4699 & 13 & 130 & 0 & 3 & 56 \\
\hline GEOM40a & 40 & 206960 & 98635 & 43 & 4 & 117 & 3 & 1 \\
\hline GEOM40b & & 275519 & 131721 & 14 & 543 & 0 & 30 & 15 \\
\hline GEOM50 & & 57869 & 27159 & 27 & 0 & 0 & 9 & 0 \\
\hline GEOM50a & 50 & 40966958 & 19591854 & 227 & 13 & 138 & 12 & 0 \\
\hline GEOM50b & & 14438962 & 6973218 & 564 & 3 & 6 & 18 & 0 \\
\hline GEOM60 & & 178478 & 82471 & 63 & 0 & 0 & 0 & 2 \\
\hline GEOM60a & 60 & 59350249 & 28292260 & 241 & 19 & 182 & 28 & 24 \\
\hline GEOM60b & & 1700740733 & 807817043 & 1691 & 25 & 176 & 23 & 0 \\
\hline GEOM70 & & 320560 & 148877 & 128 & 0 & 12 & 3 & 1 \\
\hline GEOM70a & 70 & 1662200599 & 781294815 & 881 & 6 & 192 & 20 & 0 \\
\hline GEOM70b & & 301138496 & 143985463 & 1171 & 21 & 226 & 46 & 5 \\
\hline GEOM80 & & 2173324 & 1008986 & 372 & 5 & 131 & 19 & 0 \\
\hline GEOM80a & 80 & 8859155916 & 4149659761 & 761 & 30 & 389 & 85 & 5 \\
\hline GEOM $80 b$ & & 3687195162 & 1739030200 & 480 & 29 & 303 & 138 & 0 \\
\hline GEOM90 & & 3841482 & 1748958 & 471 & 2 & 223 & 31 & 9 \\
\hline GEOM90a & 90 & 8424930433 & 3953124503 & 143 & 3 & 411 & 349 & 0 \\
\hline GEOM90b & & 6454145085 & 3036820947 & 317 & 11 & 417 & 93 & 0 \\
\hline GEOM100 & & 33141115 & 15198269 & 702 & 11 & 221 & 54 & 27 \\
\hline GEOM100a & 100 & 6622094014 & 3084753118 & 330 & 2 & 1367 & 2162 & 245 \\
\hline GEOM100b & & 5409742123 & 2511274478 & 130 & 301 & 0 & 567 & 136 \\
\hline GEOM110 & & 12496119255 & 5760860721 & 426 & 16 & 320 & 54 & 2 \\
\hline GEOM110a & 110 & 5930484572 & 2724545532 & 151 & 501 & 0 & 619 & 79 \\
\hline GEOM110b & & 4177753606 & 1922426902 & 118 & 754 & 0 & 460 & 0 \\
\hline GEOM120 & & 637959908 & 289378147 & 1015 & 19 & 357 & 128 & 8 \\
\hline GEOM120a & 120 & 5560296354 & 2542244856 & 172 & 782 & 0 & 678 & 0 \\
\hline GEOM120b & & 4003420813 & 1841115383 & 273 & 1258 & 0 & 959 & 0 \\
\hline
\end{tabular}

We also detected a mistake in [2], where the heuristic result presented in it for constraint matrix $C_{25}^{1}$ and demand vector $D_{25}^{4}$ is better (with objective function value 121) than the exact solutions obtained by both CP and IP (with objective function value 200). In fact, no other work in the literature obtained a solution with span lower than 200.

Finally, the numbers of branches and paths that do not reach a solution in the CP enumeration and generated cuts of each type in the IP enumeration are given in Tables 6 and 7 for instances with unit color demands and in Tables 8 and 9 for instances with multicoloring demands. We note that there is not a clear correlation between the instance size and this data, indicating that these parts of the algorithms are sensitive to the individual 
TABLE 7. Number of branches and fails (for CP) and of global cuts of each type (for IP) applied to MS-CAP instances (Philadelphia, Helsinki and Artificial) without multicoloring demands.

\begin{tabular}{|c|c|c|c|c|c|c|c|c|}
\hline \multirow[b]{2}{*}{ Instance } & \multirow[b]{2}{*}{$\begin{array}{l}\text { Num. } \\
\text { Vert. }\end{array}$} & \multicolumn{2}{|c|}{ Const. Prog. (CP Optimizer) } & \multicolumn{5}{|c|}{ Integer Progr. (CPLEX) } \\
\hline & & \# Branches & \# Fails & $\begin{array}{l}\# \\
\text { Clique } \\
\text { cuts }\end{array}$ & $\begin{array}{l}\# \\
\text { Implied } \\
\text { bound } \\
\text { cuts }\end{array}$ & $\begin{array}{l}\text { \# MIP } \\
\text { rounding } \\
\text { cuts }\end{array}$ & $\begin{array}{l}\text { \# Zero- } \\
\text { half cuts }\end{array}$ & $\begin{array}{l}\# \\
\text { Gomory } \\
\text { fractional } \\
\text { cuts }\end{array}$ \\
\hline$C_{21}^{1}, C_{21}^{3}, C_{21}^{5}$ and $C_{21}^{7}$ & 21 & 49177 & 24602 & 3 & 5 & 0 & 9 & 0 \\
\hline$C_{21}^{2}, C_{21}^{4}, C_{21}^{6}$ and $C_{21}^{8}$ & & 6033 & 2970 & 5 & 5 & 0 & 58 & 3 \\
\hline$C_{25}^{1}$ & 25 & 176565 & 87496 & 0 & 0 & 0 & 0 & 0 \\
\hline$C_{55}^{1}$ & 55 & 49096 & 24214 & 0 & 0 & 0 & 0 & 0 \\
\hline
\end{tabular}

TABLE 8. Number of branches and fails (for CP) and of global cuts of each type (for IP) applied to GEOM instances with multicoloring demands.

\begin{tabular}{|c|c|c|c|c|c|c|c|c|}
\hline \multirow{2}{*}{ Instance } & \multirow{2}{*}{$\begin{array}{l}\text { Num. } \\
\text { Vert. }\end{array}$} & \multicolumn{2}{|c|}{ Const. Prog. (CP Optimizer) } & \multicolumn{5}{|c|}{ Integer Progr. (CPLEX) } \\
\hline & & \# Branches & \# Fails & $\begin{array}{l}\# \\
\text { Clique } \\
\text { cuts }\end{array}$ & $\begin{array}{l}\# \text { Implied } \\
\text { bound cuts }\end{array}$ & $\begin{array}{l}\text { \# MIP } \\
\text { rounding } \\
\text { cuts }\end{array}$ & $\begin{array}{l}\text { \# Zero-half } \\
\text { cuts }\end{array}$ & $\begin{array}{l}\text { \# Gomory } \\
\text { fractional } \\
\text { cuts }\end{array}$ \\
\hline GEOM20 & & 1847656377 & 823996186 & 26 & 33 & 0 & 22 & 0 \\
\hline GEOM20a & 20 & 2060224899 & 926128135 & 35 & 378 & 0 & 92 & 91 \\
\hline GEOM $20 \mathrm{~b}$ & & 23433594 & 11236673 & 19 & 28 & 0 & 47 & 49 \\
\hline GEOM30 & & 1594445634 & 702092983 & 345 & 1 & 7 & 29 & 0 \\
\hline GEOM30a & 30 & 778845728 & 343378790 & 1234 & 6 & 189 & 3 & 0 \\
\hline GEOM30b & & 4336696005 & 2043720645 & 23 & 96 & 0 & 17 & 53 \\
\hline GEOM40 & & 1101215203 & 477955956 & 186 & 287 & 0 & 158 & 0 \\
\hline GEOM40a & 40 & 853750393 & 376905743 & 131 & 7 & 322 & 4 & 0 \\
\hline GEOM40b & & 2915214186 & 1378502269 & 361 & 1 & 4 & 2 & 0 \\
\hline GEOM50 & & 858223844 & 366277155 & 575 & 0 & 79 & 31 & 0 \\
\hline GEOM50a & 50 & 373860395 & 159472193 & 183 & 446 & 0 & 235 & 4 \\
\hline GEOM50b & & 2205618883 & 1031493835 & 1498 & 2 & 295 & 7 & 0 \\
\hline GEOM60 & & 884613825 & 374341633 & 468 & 1 & 34 & 38 & 0 \\
\hline GEOM60a & 60 & 327100218 & 135221648 & 1109 & 335 & 0 & 1213 & 0 \\
\hline GEOM60b & & 1625337918 & 741993923 & 143 & 3 & 456 & 7 & 0 \\
\hline GEOM70 & & 480579106 & 200225715 & 497 & 1363 & 0 & 275 & 0 \\
\hline GEOM70a & 70 & 178618063 & 71625162 & 0 & 0 & 0 & 0 & 0 \\
\hline GEOM70b & & 1153010252 & 520892432 & 133 & 2 & 645 & 165 & 29 \\
\hline GEOM80 & & 491579072 & 131225337 & 786 & 1842 & 0 & 1343 & 85 \\
\hline GEOM80a & 80 & 206713326 & 84260206 & 0 & 0 & 0 & 0 & 0 \\
\hline GEOM80b & & 972753631 & 434139515 & 328 & 1065 & 0 & 690 & 97 \\
\hline GEOM90 & & 289804704 & 110251804 & 1321 & 0 & 0 & 1894 & 0 \\
\hline GEOM90a & 90 & 203904382 & 82252836 & 0 & 0 & 0 & 0 & 0 \\
\hline GEOM90b & & 677312582 & 300211365 & 344 & 481 & 0 & 877 & 0 \\
\hline GEOM100 & & 219953270 & 80390988 & 0 & 0 & 0 & 0 & 0 \\
\hline GEOM100a & 100 & 134018202 & 52245146 & 0 & 0 & 0 & 0 & 0 \\
\hline GEOM100b & & 511570190 & 224573165 & 0 & 0 & 0 & 0 & 0 \\
\hline GEOM110 & & 170668054 & 64299043 & 0 & 0 & 0 & 0 & 0 \\
\hline GEOM110a & 110 & 113908674 & 41331333 & 0 & 0 & 0 & 0 & 0 \\
\hline GEOM110b & & 409339582 & 177985395 & 0 & 0 & 0 & 0 & 0 \\
\hline GEOM120 & & 207980564 & 81060477 & 0 & 0 & 0 & 0 & 0 \\
\hline GEOM120a & 120 & 82058712 & 30432596 & 0 & 0 & 0 & 0 & 0 \\
\hline GEOM120b & & 386743755 & 164997551 & 0 & 0 & 0 & 0 & 0 \\
\hline
\end{tabular}


TABLE 9. Number of branches and fails (for CP) and of global cuts of each type (for IP) applied to MS-CAP instances (Philadelphia, Helsinki and Artificial).

\begin{tabular}{|c|c|c|c|c|c|c|c|c|c|}
\hline \multirow[b]{2}{*}{$\begin{array}{l}\text { Const. } \\
\text { Matr. }\end{array}$} & \multirow[b]{2}{*}{$\begin{array}{l}\text { Demd. } \\
\text { Vect. }\end{array}$} & \multirow[b]{2}{*}{$\begin{array}{l}\text { Num. } \\
\text { Vert. }\end{array}$} & \multicolumn{2}{|c|}{ Const. Prog. (CP Optimizer) } & \multicolumn{5}{|c|}{ Integer Progr. (CPLEX) } \\
\hline & & & \# Branches & \# Fails & $\begin{array}{l}\# \\
\text { Clique } \\
\text { cuts }\end{array}$ & $\begin{array}{l}\# \\
\text { Implied } \\
\text { bound } \\
\text { cuts }\end{array}$ & $\begin{array}{l}\# \quad \text { MIP } \\
\text { rounding } \\
\text { cuts }\end{array}$ & $\begin{array}{l}\text { \# Zero- } \\
\text { half cuts }\end{array}$ & $\begin{array}{l}\text { \# Gomory } \\
\text { fractional } \\
\text { cuts }\end{array}$ \\
\hline$C_{21}^{1}$ & $D_{21}^{1}$ & \multirow{16}{*}{21} & 2555 & 504 & 0 & 0 & 0 & 0 & 0 \\
\hline$C_{21}^{1}$ & $D_{21}^{2}$ & & 469 & 4 & 0 & 0 & 0 & 0 & 0 \\
\hline$C_{21}^{2}$ & $D_{21}^{1}$ & & 6610 & 1505 & 0 & 0 & 0 & 0 & 0 \\
\hline$C_{21}^{2}$ & $D_{21}^{2}$ & & 822377 & 309790 & 0 & 0 & 0 & 0 & 0 \\
\hline$C_{21}^{3}$ & $D_{21}^{1}$ & & 2451 & 504 & 0 & 0 & 0 & 0 & 0 \\
\hline$C_{21}^{3}$ & $D_{21}^{2}$ & & 2874 & 505 & 0 & 0 & 0 & 0 & 0 \\
\hline$C_{21}^{4}$ & $D_{21}^{1}$ & & 20982 & 5811 & 0 & 0 & 0 & 0 & 0 \\
\hline$C_{21}^{4}$ & $D_{21}^{2}$ & & 44845827 & 17108454 & 0 & 0 & 0 & 0 & 0 \\
\hline$C_{21}^{5}$ & $D_{21}^{1}$ & & 2305 & 504 & 0 & 0 & 0 & 0 & 0 \\
\hline$C_{21}^{5}$ & $D_{21}^{2}$ & & 158368 & 50239 & 0 & 0 & 0 & 0 & 0 \\
\hline$C_{21}^{6}$ & $D_{21}^{1}$ & & 180812651 & 65917318 & 160 & 4 & 210 & 116 & 0 \\
\hline$C_{21}^{6}$ & $D_{21}^{2}$ & & 347622756 & 121153772 & 170 & 1 & 37 & 159 & 0 \\
\hline$C_{21}^{7}$ & $D_{21}^{1}$ & & 4106 & 505 & 0 & 0 & 0 & 0 & 0 \\
\hline$C_{21}^{7}$ & $D_{21}^{2}$ & & 352244757 & 132070514 & 4 & 0 & 0 & 8 & 35 \\
\hline$C_{21}^{8}$ & $D_{21}^{1}$ & & 199948906 & 75399919 & 163 & 1 & 379 & 224 & 0 \\
\hline$C_{21}^{8}$ & $D_{21}^{2}$ & & 451729822 & 146753868 & 225 & 204 & 0 & 190 & 0 \\
\hline$C_{25}^{1}$ & $D_{25}^{3}$ & \multirow{2}{*}{25} & 2435656356 & 1064961796 & 2 & 134 & 0 & 81 & 143 \\
\hline$C_{25}^{1}$ & $D_{25}^{4}$ & & 374343491 & 115700707 & 0 & 0 & 0 & 0 & 0 \\
\hline$C_{55}^{1}$ & $D_{55}^{5}$ & \multirow{2}{*}{55} & 7809104 & 2371461 & 0 & 0 & 0 & 0 & 0 \\
\hline$C_{55}^{1}$ & $D_{55}^{6}$ & & 27897 & 9552 & 0 & 0 & 0 & 0 & 0 \\
\hline
\end{tabular}

values of distances and demands in the instances. We also conjecture that the input graph layout also impacts in the enumeration, since some known cuts applied to coloring problems, such as clique cuts [23], are derived according to the graph to be colored.

\section{CONCLUding REMARKS}

In this paper, we addressed channel assignment in wireless networks as special graph coloring with distance constraints, and explored some feasibility properties on them, by proving some specific graph classes which admit or do not admit solutions. The special coloring problems with distance constraints were modeled by distance geometry being considered as the general problem. We have assigned the vertices on the real line, according to the distances between adjacent vertices. Beyond that, we have described feasibility conditions for some classes of graphs.

We employed constraint and integer programming formulations, which were implemented using computational OR tools, and applied them to instances from the literature in order to verify which mathematical modeling tool is best for these distance coloring models. Since the constraints from the problems are naturally transported to constraint programming, its implementation reaches the optimal solution much faster than the integer programming one for $\mathrm{BCP}$ instances. However, for BMCP, due to needing the expansion of color demands into 
additional variables, constraint programming becomes slower than integer programming, which does not need such expansion.

Ongoing and future works include improving the $\mathrm{CP}$ formulation by domain reduction and more specific constraints, and also use hybrid methods, combining both CP and IP, as well as heuristics, in order to solve the distance coloring models faster. The study of the problems posed to specific classes of graphs, in order to establish the characterization of feasibility conditions for them, is subject of the research in progress.

Acknowledgements. Supported by CAPES (Coordenação de Aperfeiçoamento de Pessoal de Ensino Superior), CNPq (Conselho Nacional de Desenvolvimento Científico e Tecnológico), FAPEAM (Fundação de Amparo a Pesquisa do Estado do Amazonas) and Méliuz - Brazil.

\section{REFERENCES}

[1] G. Audhya, K. Sinha, S. Ghosh and B. Sinha, A survey on the channel assignment problem in wireless networks. Wireless Commun. Mobile Comput. 11 (2011) 583-609.

[2] G. Chakraborty, An efficient heuristic algorithm for channel assignment problem in cellular radio networks. IEEE Trans. Veh. Technol. 50 (2001) 1528-1539.

[3] D. Costa, On the use of some known methods for T-coloring of graphs. Ann. Oper. Res. 41 (1999) 343-358.

[4] R. de Freitas, B. Dias, N. Maculan and J. Szwarcfiter, Distance geometry approach for special graph coloring problems. Preprint arXiv: $1606.04978(2016)$.

[5] R. de Freitas, B. Dias, N. Maculan and J. Szwarcfiter, On distance graph coloring problems. Int. Trans. Oper. Res. (2019). DOI: 10.1111 /itor.12626.

[6] B. Dias, Theoretical models and algorithms for optimizing channel assignment in wireless mobile networks. Master's thesis, Universidade Federal do Amazonas, Brazil (2014). In Portuguese.

[7] B. Dias, R. de Freitas, N. Maculan and P. Michelon, Solving the bandwidth coloring problem applying constraint and integer programming techniques. Optim. Online (e-print) (2016). Available at http://www.optimization-online.org/DB_HTML/2016/ 06/5514.html.

[8] R. Dorne and J. Hao, Tabu search for graph coloring, T-coloring and set T-colorings, edited by S. Voss, S. Martello, I.H. Osman and C. Roucairol. In: Metaheuristics: Advances and Trends in Local Search Paradigms for Optimization. Springer, New York, NY (1999) 77-92.

[9] P. Galinier and A. Hertz, A survey of local search methods for graph coloring. Comput. Oper. Res. 33 (2006) $2547-2562$.

[10] M. Garey and D. Johnson, Computers and Intractability: A Guide to the Theory of NP-Completeness. W.H. Freeman \& Co, New York, NY (1979).

[11] A.I. Giortzis and L.F. Turner, Application of mathematical programming to the fixed channel assignment problem in mobile radio networks. IEE Proc. Commun. 144 (1997) 257-264.

[12] A. Gueham, A. Nagih, H.A. Haddadene and M. Masmoudi, Graph coloring approach with new upper bounds for the chromatic number: team building application. RAIRO:OR 52 (2018) 807-818.

[13] W. Hale, Frequency assignment: theory and applications. Proc. IEEE 25 (1980) 1497-1514.

[14] R. Karp, Reducibility among combinatorial problems, edited by R.E. Miller and J.W. Thatcher. In: Complexity of Computer Computations. Plenum Press, New York, NY (1972) 85-103.

[15] G. Kendall and M. Mohamad, Solving the fixed channel assignment problem in cellular communications using an adaptive local search, edited by E. Burke and M. Trick. In: Vol. 3616 of 5th International Conference for the Practice and Theory of Automated Timetabling (PATAT 2004). Lecture Notes in Computer Science. Springer, Heidelberg (2005).

[16] S. Kim, A.E. Smith and J. Lee, A memetic algorithm for channel assignment in wireless fdma systems. Comput. Oper. Res. 34 (2007) 1842-1856.

[17] A. Koster, Frequency assignment: models and algorithms. Ph.D. thesis, Universiteit Maastricht (1999).

[18] X. Lai and Z. Lü, Multistart iterated tabu search for bandwidth coloring problem. Comput. Oper. Res. 40 (2013) $1401-1409$.

[19] V. Mak, Polyhedral studies for minimum-span graph labelling with integer distance constraints. Int. Trans. Oper. Res. 14 (2007) 105-121.

[20] E. Malaguti and P. Toth, An evolutionary approach for bandwidth multicoloring problems. Eur. J. Oper. Res. 189 (2008) 638-651.

[21] E. Malaguti and P. Toth, A survey on vertex coloring problems. Int. Trans. Oper. Res. 17 (2010) 1-34.

[22] A. Mehrotra and M. Trick, A branch-and-price approach for graph multi-coloring, edited by E.K. Baker, A. Joseph, A. Mehrotra and M. Trick. In: Vol. 37 of Extending the Horizons: Advances in Computing, Optimization, and Decision Technologies. Operations Research/Computer Science Interfaces Series. Springer, New York, NY (2007) 15-29.

[23] I. Méndez-Díaz and P. Zabala, A Branch-and-Cut algorithm for graph coloring. Dis. Appl. Math. 154 (2006) $826-847$.

[24] V. Phan and S. Skiena, Coloring graphs with a general heuristic search engine. In: Proceedings of the Computational Symposium on Graph Coloring and Its Generalizations. Ithaca, NY (2002) 92-99. 
[25] J.B. Saxe, Embeddability of weighted graphs in $k$-space is strongly NP-hard. In: Proceedings of 17th Allerton Conference in Communications, Control and Computing. (1979) 480-489.

[26] M. Trick, A. Mehrotra, and D. Johnson. COLOR02/03/04: Graph coloring and its generalizations. In: Proceedings of the Computational Symposium on Graph Coloring and Its Generalizations. Ithaca, NY (2002).

[27] S. Varone and N. Zufferey, Three tabu search methods for the MI-FAP applied to 802.11 networks. RAIRO:OR 42 (2008) 501-514.

[28] M. Vasquez, A. Dupont and D. Habet, Consistency checking within local search applied to the frequency assignment with polarization problem. RAIRO:OR 37 (2003) 311-323. 